\title{
COUNTING CYCLES IN PERMUTATIONS BY GROUP CHARACTERS, WITH AN APPLICATION TO A TOPOLOGICAL PROBLEM
}

\author{
D. M. JACKSON
}

\begin{abstract}
The character theory of the symmetric group is used to derive properties of the number of permutations, with $k$ cycles, which are expressible as the product of a full cycle with an element of an arbitrary, but fixed, conjugacy class. For the conjugacy class of fixed point free involutions, this problem has application to the analysis of singularities in surfaces.
\end{abstract}

1. Introduction. For nonnegative integers $k$ and $\mathbf{N}$, and a partition $\psi$ of $\mathbf{N}$, let $e_{k}^{\psi}$ denote the number of permutations $\pi$ on $\mathbf{N}$ symbols such that $\pi$ has exactly $k$ cycles, and such that $\pi$ can be expressed as a product of an arbitrary, but fixed, cycle of length $\mathbf{N}$ and a permutation in the conjugacy class indexed by $\psi$. The purpose of this paper is to derive the generating function for these numbers, and to obtain some of their properties. The method makes direct use of combinatorial and algebraic properties of the group algebra of the symmetric group.

A special case of this problem is of particular interest. Let $e_{k}^{(p)}(n)$ denote the number $e_{k}^{\psi}$ when $\psi$ indexes the conjugacy class of permutations on $p n$ symbols, with $n$ cycles of length $p$. The matter of calculating $e_{k}^{(2)}(n)$ arose in connection with work by Harris and Morrison [4] on singularities in surfaces. It has also occurred indirectly in the work of Gross [2] on graph embeddings. Harer and Zagier [3] have shown, by an independent method, that the sequence $e_{k}^{(2)}(n)$ for $k, n \geqslant 1$ satisfies a three-term linear recurrence equation with coefficients which are polynomials in $n$.

To fix ideas, note that for $n=2$ the permissible permutations are

$$
\{(1234)(12)(34),(1234)(13)(24),(1234)(14)(23)\}=\{(13),(1432),(24)\}
$$

so

$$
e_{1}^{(2)}(2)=|\{(1432)\}|=1, \quad e_{2}^{(2)}(2)=0, \quad e_{3}^{(2)}(2)=|\{(13),(24)\}|=2 .
$$

Values of $e_{k}^{(2)}(n)$ for some other $(k, n)$ are given in Table $\mathrm{I}$ of the Appendix.

The combinatorial results involving the use of the group algebra of the symmetric group $\mathbf{S}_{n}$ on $n$ symbols over $\mathbf{C}$ are given in $\$ 2$ together with the appropriate facts about orthogonal idempotents in the center of the group algebra. The results which we need about specific characters of $\mathbf{S}_{n}$ are derived in $\$ 3$ from Frobenius theory. Although these results are known, uniform proofs of them are not readily available

Received by the editors October 11, 1985.

1980 Mathematics Subject Classification (1985 Revision). Primary 05A15, 20C15; Secondary 57N37. 
and they have therefore been included here. The generating function for $e_{k}^{\psi}$ is derived in $\$ 4$ by evaluating certain character sums. It is stated in Theorem 4.5 . An explicit expression for $e_{k}^{(p)}(n)$ is given in terms of standard combinatorial numbers in $\S 5$, and in $\S 6$ we show that $e_{k}^{(2)}(n)$ and $e_{k}^{(3)}(n)$ satisfy linear recurrence equations with polynomial coefficients. We also show that

$$
e_{n+1-2 r}^{(2)}(n)=\frac{1}{2}\left(\begin{array}{c}
2 n \\
n
\end{array}\right)\left(\begin{array}{c}
n+1 \\
2 r+1
\end{array}\right) \rho_{r}(n) /(r+1)(n+1),
$$

where $\rho_{r}(n)$ is a polynomial of degree $r-1$ in $n$. In $\S 7$ we use the form of the generating function for $e_{k}^{\gamma}$ to establish a bijection between an easily enumerated set of permutations and another set directly related to the permutations counted by $e_{k}^{\gamma}$. It would be of considerable interest to establish this bijection combinatorially, and, by so doing, provide a combinatorial proof of the expression giving $e_{k}^{\gamma}$.

The group algebra of $\mathbf{S}_{n}$ has been used by Stanley [7] in connection with factorizing permutations into cycles of length $n$. Character theoretic methods have been applied by Thompson [8] to a problem which can be stated enumeratively.

The following notation is needed. A partition $\rho=\left(\rho_{1}, \rho_{2}, \ldots\right)$ is a sequence (finite or infinite) of nonnegative integers such that $\rho_{1} \geqslant \rho_{2} \geqslant \cdots$. The nonzero elements of $\rho$ are called parts, the number of parts is the length, $l(\rho)$, of $\rho$ and the sum of all parts is the weight, $|\rho|$, of $\rho$. If $\rho$ is a partition of weight $\mathbf{N}$ we write $\rho \vdash \mathbf{N}$. Let $m_{j}$ be the multiplicity of $j$ in $\rho$ for $j \geqslant 1$. We write $\rho=\left\langle m_{1}, m_{2}, \ldots\right\rangle$ or, equivalently, $\rho=\left[1^{a_{1}} 2^{a_{2}} \ldots\right]$ with the convention that $j^{m_{j}}$ is omitted if $m_{j}=0$, and $j^{m_{j}}$ is replaced by $j$ if $m_{j}=1$. We also adopt the convention that $\left[1,1^{i}\right]=\left[1^{i}, 1\right]=\left[1^{i+1}\right]$. For example $(5,2,2)=\left[2^{2} 5\right]=\langle 0,2,0,0,1\rangle \vdash 9$ and $l\left(\left[2^{2} 5\right]\right)=3$. Let $\alpha=\left[1^{a_{1}} 2^{a_{2}}\right.$ $\cdots], \beta=\left[1^{b_{1}} 2^{b_{2}} \cdots\right]$. Then $\alpha \geqslant \beta$ if and only if $a_{i} \geqslant b_{i}$ for all $i \geqslant 1$, and $\alpha=\beta$ if and only if $a_{i}=b_{i}$ for all $i \geqslant 1$. The sum of $\alpha$ and $\beta$ is $\alpha+\beta=\left[1^{a_{1}+b_{1}} 2^{a_{2}+b_{2}} \ldots\right]$. Let $\delta_{\alpha \beta}=1$ if $\alpha=\beta$ and $\delta_{\alpha \beta}=0$ if $\alpha \neq \beta$.

Each element of the symmetric group $\pi \in \mathbf{S}_{\mathbf{N}}$ of all permutations on the set $[\mathbf{N}]=\{1, \ldots, \mathbf{N}\}$ can be expressed uniquely (up to order) as a product of disjoint cycles. The number of such cycles is denoted by $\kappa(\pi)$. The cycle $\sigma$ such that $\sigma i_{j}=i_{j+1}$ for $1 \leqslant j<k, \sigma i_{k}=i_{1}$, where $i_{1}, \ldots, i_{k} \in[\mathbf{N}]$, is denoted by $\left(i_{1}, \ldots, i_{k}\right)$. The cycle $(1,2, \ldots, \mathbf{N})$ is denoted by $\omega_{N}$. The cycle-type of $\pi$ is $\tau(\pi)=\left[1^{j_{1}} 2^{j_{2}}\right.$ $\cdots \mathbf{N}^{j_{N}}$ ], where $\pi$ has $j_{k}$ cycles of length $k$ for $1 \leqslant k \leqslant \mathbf{N}$. A cycle of length $j$ is called a $j$-cycle, and we adopt the convention that 1-cycles are omitted from the disjoint cycle representation of $\pi$. Thus, if $\left\{i_{1}, \ldots, i_{k}\right\} \in[\mathbf{N}]$, then $\left(i_{1}, \ldots, i_{k}\right)$ denotes either a $k$-cycle or an element of $\mathbf{S}_{\mathbf{N}}$ of type $\left[1^{n-k} k\right]$. The distinction between these will be clear from the context. The multiplication of permutations is carried out from right to left. Thus if $\pi=\omega_{6}(12)(36)(45)$, then $\pi=(13)(46)$ so $\tau(\pi)=\left[1^{2} 2^{2}\right]$ and $\kappa(\pi)=4$. Finally, if $w_{1}, \ldots, w_{k}$ are indeterminates and $\mathbf{i}=$ $\left(i_{1}, \ldots, i_{k}\right)$ is a vector of integers, then the monomial $w_{1}^{i_{1}} w_{2}^{i_{2}} \cdots w_{k}^{i_{k}}$ is denoted by $\mathbf{w}^{\mathbf{i}}$.

2. Combinatorial lemmas and the group algebra $\mathbf{C S}_{\mathbf{N}}$. Each element $a$ of the group algebra $\mathbf{C S}_{\mathbf{N}}$ can be expressed as $\sum_{\sigma \in \mathbf{S}_{\boldsymbol{N}}} a_{\sigma} \boldsymbol{\sigma}$, where $a_{\sigma} \in \mathbf{C}$. The product $a b$ of $a, b \in \mathbf{C S}_{\mathbf{N}}$ is defined to be $\sum_{\sigma, \sigma^{\prime} \in \mathbf{S}_{\mathbf{N}}} a_{\sigma} b_{\sigma^{\prime}} \sigma \sigma^{\prime} \in \mathbf{C S}_{\mathbf{N}}$, where of course $\sigma \sigma^{\prime} \in \mathbf{S}_{\mathbf{N}}$. 
For $\theta \vdash \mathbf{N}$ let $\mathbf{K}_{\theta}=\sum_{g \in C_{\theta}} g$, where $C_{\theta}=\left\{\pi \in \mathbf{S}_{\mathbf{N}} \mid \tau(\pi)=\boldsymbol{\theta}\right\}$ is a conjugacy class of $\mathbf{S}_{\mathbf{N}}$, each element of which has $l(\theta)$ cycles. Then $\left\{K_{\theta} \mid \theta \vdash \mathbf{N}\right\}$ is a basis of the center $\mathbf{Z}$ of $\mathbf{C S}_{\mathbf{N}}$, so the coefficient operator

$$
\left[\mathbf{K}_{\alpha}\right]: \mathbf{Z} \rightarrow \mathbf{C}: \sum_{\theta \vdash \mathbf{N}} c_{\theta} \mathbf{K}_{\theta} \mapsto c_{\alpha}
$$

is well defined and acts linearly on $\mathbf{Z}$. The following results are important combinatorially.

Proposition 2.1. Let $\alpha, \beta, \gamma \vdash \mathbf{N}$, and let $c, c^{\prime} \in C_{\gamma}$. Then

$$
\left|\left\{(a, b) \in C_{\alpha} \times C_{\beta} \mid a b=c\right\}\right|=\left|\left\{(a, b) \in C_{\alpha} \times C_{\beta} \mid a b=c^{\prime}\right\}\right| .
$$

Proposition 2.2. If $\alpha, \beta, \theta \vdash \mathbf{N}$, then the number of ways of expressing $c \in C_{\theta}$ as $c=a b$ with $(a, b) \in C_{\alpha} \times C_{\beta}$ is $\left[\mathbf{K}_{\theta}\right] \mathbf{K}_{\alpha} \mathbf{K}_{\beta}$.

We may now give an expression for $e_{k}^{\gamma}$ in terms of the group algebra.

LemMA 2.3. Let $e^{\gamma}=\left|\omega_{\mathrm{N}} C_{\gamma} \cap C_{\theta}\right|$, where $\theta \vdash \mathbf{N}$. Then

(i)

$$
e_{k}^{\gamma}=\sum_{\substack{\theta \vdash \mathbf{N} \\ l(\theta)=k}} e_{\theta}^{\gamma}
$$

(ii)

$$
e_{\theta}^{\gamma}=\frac{h^{\theta}}{h^{[\mathbf{N}]}}\left[\mathbf{K}_{\theta}\right] \mathbf{K}_{[\mathbf{N}]} \mathbf{K}_{\gamma}, \quad \text { where } h^{\theta}=\left|C_{\theta}\right|
$$

Proof. (i) Immediate.

(ii) By definition

$$
e_{\theta}^{\gamma}=\left|\omega_{\mathbf{N}} C_{\gamma} \cap C_{\theta}\right|=\sum_{\substack{(a, b) \in\left\{\omega_{\mathbf{N}}\right\} \times C_{\gamma} \\ a b \in C_{\theta}}}=\frac{1}{h^{[\mathbf{N}]}} \sum_{\substack{(a, b) \in C_{[N]} \times C_{\gamma} \\ a b \in C_{\theta}}},
$$

so from Proposition 2.1

$$
e_{\theta}^{\gamma}=\frac{h^{\theta}}{h^{[\mathbf{N}]}} \sum_{\substack{(a, b) \in C_{[\mathbf{N}]} \times C_{\gamma} \\ a b=c}}
$$

for an arbitrary $c \in C_{\theta}$. But, from Proposition 2.2

$$
\sum_{\substack{(a, b) \in C_{[\mathbf{N}]} \times C_{\gamma} \\ a b=c}}=\left[\mathbf{K}_{\theta}\right] \mathbf{K}_{[\mathbf{N}]} \mathbf{K}_{\gamma}
$$

and the result follows.

To evaluate $\left[\mathbf{K}_{\theta}\right] \mathbf{K}_{\alpha} \mathbf{K}_{\beta}$ for arbitrary $\alpha, \beta, \theta \vdash \mathbf{N}$, we recall (Burrow [1]) that $\mathbf{C S}_{\mathbf{N}}$ is semisimple, so $\mathbf{Z}$, the center of $\mathbf{C S}_{\mathbf{N}}$, has a basis consisting of orthogonal idempotents. Let $\chi^{\theta}$ be the irreducible (ordinary) character associated with $C_{\theta}$ and let $f^{\theta}$ be the degree of $\chi^{\theta}$. The value of $\chi^{\theta}$ at any element of $C_{\alpha}$ is denoted by $\chi_{\alpha}^{\theta}$, and $f^{\theta}=\chi_{\left[1^{\mathrm{N}}\right]}^{\theta}$. 
LEMMA 2.4. Let $\alpha \vdash \mathbf{N}$, and

$$
F_{\alpha}=\frac{f^{\alpha}}{\mathbf{N} !} \sum_{\theta \vdash \mathbf{N}} \chi_{\theta}^{\alpha} \mathbf{K}_{\theta}
$$

Then $\left\{\mathbf{F}_{\alpha} \mid \alpha \vdash \mathbf{N}\right\}$ is a basis of $\mathbf{Z}$ consisting of orthogonal idempotents (i.e. $\mathbf{F}_{\alpha} \mathbf{F}_{\beta}=$ $\left.\mathbf{F}_{\alpha} \delta_{\alpha \beta}\right)$. Moreover

$$
\mathbf{K}_{\alpha}=h^{\alpha} \sum_{\theta \vdash N} \frac{1}{f^{\theta}} \chi_{\alpha}^{\theta} \mathbf{F}_{\theta} .
$$

It is now possible to express $e_{k}^{\gamma}$ as a character sum.

Corollary 2.5. Let $\psi \vdash \mathbf{N}$. Then

$$
e_{\psi}^{\gamma}=\frac{h^{\gamma} h^{\psi}}{\mathbf{N} !} \sum_{\theta \vdash \mathbf{N}} \frac{1}{f^{\theta}} \chi_{[\mathbf{N}]}^{\theta} \chi_{\gamma}^{\theta} \chi_{\psi}^{\theta}
$$

Proof. From Lemmas 2.3(ii) and 2.4

$$
e_{\psi}^{\gamma}=\frac{h^{\psi}}{h^{[\mathbf{N}]}}\left[\mathbf{K}_{\psi}\right] \mathbf{K}_{[\mathbf{N}]} \mathbf{K}_{\gamma}=h^{\psi} h^{\gamma} \sum_{\alpha, \beta \vdash \mathbf{N}}\left(f^{\alpha} f^{\beta}\right)^{-1} \chi_{[\mathbf{N}]}^{\alpha} \chi_{\gamma}^{\beta}\left[\mathbf{K}_{\psi}\right] \mathbf{F}_{\alpha} \mathbf{F}_{\beta} .
$$

But $\left[\mathbf{K}_{\psi}\right] \mathbf{F}_{\alpha} \mathbf{F}_{\beta}=\delta_{\alpha \beta}\left[\mathbf{K}_{\psi}\right] \mathbf{F}_{\alpha}=\delta_{\alpha \beta} f^{\alpha} \chi_{\psi}^{\alpha} / \mathbf{N}$ ! since $\mathbf{F}_{\alpha}, \mathbf{F}_{\beta}$ are orthogonal idempotents and the result follows.

3. Character sums. In evaluating the character sum given in Corollary 2.5, we use known expressions for $\chi_{\alpha}^{\beta}$ for particular choices of $\alpha$ and $\beta$. The proofs are obtained directly from Frobenius theory and are included partly for completeness, and partly because they are not easy to extract from the literature.

If $A$ is an $r \times s$ matrix whose $(i, j)$-element is $a_{i j}$, we write $A=\left[a_{i j}\right]_{r \times s}$. When $r=s$, the determinant of $A$ is denoted by $\left\|a_{i j}\right\|$ (or by $\left\|\left[a_{i j}\right]\right\|_{r \times r}$ ).

DeFinition 3.1. Let $x_{1}, \ldots, x_{\mathbf{N}}$ be commutative indeterminates and let $\mu=$ $\left(\mu_{1}, \mu_{2}, \ldots\right) \vdash \mathbf{N}$, where $l(\mu)=m$. The power, elementary, complete and Schur symmetric functions are, respectively,

(i) $p_{\mu}=p_{\mu_{1}} p_{\mu_{2}} \cdots ; p_{i}=x_{1}^{i}+\cdots+x_{\mathrm{N}}^{i}$,

(ii) $e_{\mu}=e_{\mu_{2}} e_{\mu_{2}} \cdots ; \sum_{i=0}^{\mathbf{N}} e_{i} t^{i}=\prod_{i=1}^{\mathbf{N}}\left(1+x_{i} t\right)$,

(iii) $h_{\mu}=h_{\mu_{1}} h_{\mu_{2}} \cdots ; \sum_{i \geqslant 0} h_{i} t^{i}=\prod_{i=1}^{\mathbf{N}}\left(1-x_{i} t\right)^{-1}$

(iv) $s_{\mu}=\left\|x_{j}^{\mu_{i}+m-i}\right\| /\left\|x_{j}^{m-i}\right\|$.

Clearly, for partitions $\alpha$ and $\beta, p_{\alpha} p_{\beta}=p_{\alpha+\beta}$. Analogous statements hold for $e_{\mu}$ and $h_{\mu}$.

Proposition 3.2. Let $g(\mu)=\prod_{i}\left(i^{m_{\imath}} m_{i} !\right)^{-1}$, where $\mu=\left[1^{m_{1}} 2^{m_{2}} \cdots\right] \vdash \mathbf{N}$. Then

(i) $h^{\mu}=\mathbf{N} ! g(\mu)$.

Let $\alpha=\left[1^{a_{1}} 2^{a_{2}} \cdots\right]$ and $\beta=\left[1^{b_{1}} 2^{b_{2}} \cdots\right]$. Then

(ii) $g(\alpha) g(\beta)=g(\alpha+\beta) \prod_{i}\left(\begin{array}{c}a_{i}+b_{i} \\ a_{1}\end{array}\right)$.

Proof. Straightforward. 
By considering the expansions of

$$
\exp \log \prod_{i=1}^{\mathbf{N}}\left(1+x_{i} t\right) \text { and } \exp \log \prod_{i=1}^{\mathbf{N}}\left(1-x_{i} t\right)^{-1}
$$

it is possible to expand $e_{r}$ and $h_{r}$ in terms of power sums.

Proposition 3.3. For $r \geqslant 0$

(i) $e_{r}=\sum_{\alpha \vdash r}(-1)^{a_{2}+a_{4}+a_{6}+\cdots} g(\alpha) p_{\alpha}$, where $\alpha=\left[1^{a_{1}} 2^{a_{2}} \cdots\right]$,

(ii) $h_{r}=\sum_{\alpha \vdash r} g(\alpha) p_{\alpha}$.

The next result is a fundamental one, due to Frobenius [5], which gives the Schur functions in terms of the power sums.

TheOREM 3.4. Let $\mu \vdash \mathbf{N}$. Then $s_{\mu}=\Sigma_{\alpha \vdash \mathrm{N}} g(\alpha) \chi_{\alpha}^{\mu} p_{\alpha}$.

Let $R$ be a ring and let $\left\{B_{1}, B_{2} \cdots\right\}$ be a basis of a subring $\mathbf{B}$ of $R\left[\left[x_{1}, \ldots, x_{\mathbf{N}}\right]\right]$. The coefficient operator, []$_{\mathbf{B}}$, on $\mathbf{B}$ is defined by

$$
\left[B_{k}\right]_{\mathbf{B}}: \mathbf{B} \rightarrow \mathbf{R}: \sum_{i \geqslant 1} b_{i} B_{i} \mapsto b_{k},
$$

which acts linearly on $\mathbf{B}$. In general, $\mathbf{B}$ will be understood from the context, so the subscript $\mathbf{B}$ will be omitted from the coefficient operator. In this notation we therefore have

$$
\chi_{\alpha}^{\mu}=g^{-1}(\alpha)\left[p_{\alpha}\right] s_{\mu}
$$

as an expression for the value of $\chi^{\mu}$ on the conjugacy class $C_{\alpha}$.

A result of Jacobi expresses Schur functions in terms of complete symmetric functions.

THEOREM 3.5. Let $\mu=\left(\mu_{1}, \mu_{2}, \ldots\right)$ and $l(\mu)=m$. Then $s_{\mu}=\left\|h_{\mu_{i}-i+j}\right\|_{m \times m}$.

These results are sufficient to enable us to evaluate the required characters.

COROLlaRY 3.6. Let $\alpha=\left[1^{a_{1}} 2^{a_{2}} \cdots\right] \vdash \mathbf{N}$. Then

(i) $\chi_{\alpha}^{[\mathrm{N}]}=1$,

(ii) $\chi_{\alpha}^{\left[\left[^{\mathrm{N}}\right]\right.}=(-1)^{a_{2}+a_{4}+a_{6}+\cdots}$.

Proof. (i) follows from Theorem 3.4, Proposition 3.3(ii) and Theorem 3.5.

(ii) From Theorem 3.4

$$
\begin{aligned}
\chi_{\alpha}^{\left[\left[^{N}\right]\right.} & =g^{-1}(\alpha)\left[p_{\alpha}\right]\left\|x_{j}^{1+\mathbf{N}-i}\right\| /\left\|x_{n}^{\mathbf{N}-i}\right\|=g^{-1}(\alpha)\left[p_{\alpha}\right]\left(x_{1} \cdots x_{\mathbf{N}}\right) \\
& =g^{-1}(\alpha)\left[p_{\alpha}\right] e_{\mathbf{N}} \quad \text { (from Definition 3.1(ii)). }
\end{aligned}
$$

The result follows from Proposition 3.3(i).

Corollary 3.7.

$$
\chi_{[\mathbf{N}]}^{\alpha}= \begin{cases}(-1)^{k} & \text { if } \alpha=\left[1^{k}, \mathbf{N}-k\right] \text { for some } k, 0 \leqslant k \leqslant \mathbf{N}-1, \\ 0 & \text { otherwise }\end{cases}
$$


Proof. From Theorems 3.4 and 3.5

$$
\chi_{[\mathbf{N}]}^{\alpha}=g^{-1}([\mathbf{N}])\left[p_{N}\right]\left\|h_{\alpha_{t}-i+j}\right\|_{m \times m},
$$

where $m=l(\alpha)$ and $\alpha=\left(\alpha_{1}, \alpha_{2}, \ldots\right)$. The largest value of $\alpha_{i}-i+j$ occurs at $(i, j)=(1, m)$. From Proposition 3.3(ii), $h_{r}$ contains sums and products of $p_{1}, \ldots, p_{r}$, so $p_{\mathbf{N}}$ occurs in the determinant if and only if $\alpha_{1}-1+m \geqslant \mathbf{N}$. On the other hand, by considering the cofactor expansion of the determinant by the first column, we see that $p_{\mathbf{N}}$ occurs itself as a term if and only if $\alpha_{1}-1+m=\mathbf{N}$. Thus $\alpha_{1}=\mathbf{N}-m+1$ whence $\alpha_{2}=\cdots=\alpha_{m}=1$, since the matrix is $m \times m$. It follows that

$$
\chi_{[\mathbf{N}]}^{\alpha}=\chi_{[\mathbf{N}]}^{\left[1^{m-1}, \mathbf{N}-m+1\right]} \delta_{\alpha,\left[1^{m-1}, \mathbf{N}-m+1\right]} .
$$

But

$$
\chi_{[\mathbf{N}]}^{\left[1^{m-1}, \mathbf{N}-m+1\right]}=g^{-1}([\mathbf{N}])\left[p_{\mathbf{N}}\right](-1)^{m+1} h_{\mathbf{N}}
$$

by the cofactor expansion of $\left[h_{\alpha_{i}-i+j}\right]_{m \times m}$ for $\alpha=\left[1^{m-1}, \mathbf{N}-m+1\right]$, by the first row. From Proposition 3.3(ii), $\left[p_{\mathbf{N}}\right] h_{\mathbf{N}}=g([\mathbf{N}])$ and the result follows, since $1 \leqslant m$ $=\mathbf{N}$.

COROLlaRY 3.8. Let $\alpha=\left[1^{a_{1}} 2^{a_{2}} \cdots\right] \vdash \mathbf{N}$. Then

$$
\sum_{r=0}^{\mathbf{N}-1} \chi_{\alpha}^{\left[1^{r}, \mathbf{N}-r\right]} y^{r}=(1+y)^{-1} \prod_{i=1}^{\mathbf{N}}\left\{1-(-y)^{i}\right\}^{a_{i}} .
$$

Proof. Let $\lambda^{(r)}=\left(\lambda_{1}^{(r)}, \lambda_{2}^{(r)}, \ldots\right)=\left[1^{r}, \mathbf{N}-r\right]$. Then from Theorems 3.4 and 3.5

$$
\chi_{\alpha}^{\lambda^{(r)}}=g^{-1}(\alpha)\left[p_{\alpha}\right] s_{\lambda^{(r)}}=g^{-1}(\alpha)\left[p_{\alpha}\right]\left\|h_{\lambda_{i}^{(r)}-i+j}\right\| .
$$

Expanding the determinant by its first column gives

$$
\chi_{\alpha}^{\lambda^{(r)}}=g^{-1}(\alpha)\left[p_{\alpha}\right]\left\{h_{\mathbf{N}-r} s_{\left[1^{r}\right]}-s_{\lambda^{(r-1)}}\right\} .
$$

But $g^{-1}(\alpha)\left[p_{\alpha}\right] s_{\lambda^{(r-1)}}=\chi_{\alpha}^{\lambda^{(r-1)}}$ from Theorem 3.4. Moreover, from Proposition 3.3(ii)

$$
\begin{aligned}
g^{-1}(\alpha)\left[p_{\alpha}\right] h_{\mathbf{N}-r} s_{\left[1^{r}\right]}=g^{-1}(\alpha)\left[p_{\alpha}\right] \sum_{\beta \vdash \mathbf{N}-r} g(\beta) p_{\beta} s_{\left[1^{r}\right]} \\
=\sum_{\substack{\beta \vdash \mathbf{N}-r \\
\beta \leqslant \alpha}} \prod_{i \geqslant 1}\left(\begin{array}{c}
a_{i} \\
a_{i}-b_{i}
\end{array}\right) g^{-1}(\alpha-\beta)\left[p_{\alpha-\beta}\right] s_{\left[1^{r}\right]}
\end{aligned}
$$

(from Proposition 3.2(iii)

where $\left.\alpha=\left[1^{a_{1}} 2^{a_{2}} \cdots\right], \beta=\left[1^{b_{1}} 2^{b_{2}} \cdots\right]\right)$

$=\sum_{\rho \vdash r} \prod_{i \geqslant 1}\left(\begin{array}{l}a_{i} \\ r_{i}\end{array}\right) \chi_{\rho}^{\left[1^{r}\right]} \quad\left(\right.$ from Theorem 3.4, where $\left.\rho=\left[1^{r_{1}} 2^{r_{2}} \cdots\right]\right)$.

Combining these facts and using Corollary 3.6(ii) we have

$$
\chi_{\alpha}^{\lambda^{(r)}}=\sum_{\rho \vdash r}(-1)^{r_{2}+r_{4}+r_{6}+\cdots} \prod_{i \geqslant 1}\left(\begin{array}{l}
a_{i} \\
r_{i}
\end{array}\right)-\chi_{\alpha}^{\lambda^{(r-1)}} .
$$


Multiply both sides by $y^{r}$ and sum over $1 \leqslant r \leqslant \mathbf{N}-1$ to get

$$
C_{\mathbf{N}}(y)-\chi_{\alpha}^{[\mathbf{N}]}=u(y)-1-y^{\mathbf{N}}\left[y^{\mathbf{N}}\right] u(y)-y C_{\mathbf{N}}(y)+y^{\mathbf{N}} \chi_{\alpha}^{\left[1^{\mathbf{N}}\right]},
$$

where $C_{\mathbf{N}}(y)=\sum_{r=0}^{\mathbf{N}-1} \chi_{\alpha}^{\lambda^{(r)}} y^{r}$ and $u(y)=\prod_{i=1}^{\mathbf{N}}\left\{1-(-y)^{i}\right\}^{a_{i}}$. But $\left[y^{\mathbf{N}}\right] u(y)=$ $(-1)^{a_{2}+a_{4}+a_{6}+\cdots}$ so, from Corollary 3.6(i), (ii), $(1+y) C_{\mathrm{N}}(y)=u(y)$ and the result follows.

CoROllaRY 3.9. $f^{\left[1^{r}, \mathbf{N}-r\right]}=\left(\begin{array}{c}\mathbf{N}-1 \\ r\end{array}\right)$.

PRoof. $f^{\left[1^{r}, \mathbf{N}-r\right]}=\chi_{\left[1^{r}\right]}^{\left[r^{r} \mathbf{N}-r\right]}=\left[y^{r}\right](1+y)^{\mathbf{N}-1}$ from Corollary 3.8 and the result follows.

4. The generating function for $e_{k}^{\gamma}$. We begin by expressing $e_{\psi}^{\gamma}$ in terms of characters associated with conjugacy classes of cycles.

Proposition 4.1. Let $\psi, \gamma \vdash \mathbf{N}$. Then

$$
e_{\psi}^{\gamma}=\frac{h^{\psi} h^{\gamma}}{\mathbf{N} !} \cdot \sum_{j=0}^{\mathbf{N}-1}(-1)^{j}\left(\begin{array}{c}
\mathbf{N}-1 \\
j
\end{array}\right)^{-1} \chi_{\gamma}^{\left[1^{j}, \mathbf{N}-j\right]} \chi_{\psi}^{\left[1^{1}, \mathbf{N}-j\right]}
$$

Proof. From Corollary 2.5

$$
e_{\psi}^{\gamma}=\frac{h^{\gamma} h^{\psi}}{\mathbf{N} !} \sum_{\theta \vdash \mathbf{N}} \frac{1}{f^{\theta}} \chi_{[\mathbf{N}]}^{\theta} \chi_{\gamma}^{\theta} \chi_{\psi}^{\theta}
$$

so from Corollary 3.7

$$
e_{\psi}^{\gamma}=\frac{h^{\gamma} h^{\psi}}{\mathbf{N} !} \sum_{j=0}^{\mathbf{N}-1} \frac{(-1)^{j}}{f^{\left[1^{j}, \mathbf{N}-j\right]}} \chi_{\gamma}^{\left[1^{j}, \mathbf{N}-j\right]} \chi_{\psi}^{\left[1^{j}, \mathbf{N}-j\right]} .
$$

The result follows from Corollary 3.9.

The character sum given in Proposition 4.1 may be evaluated by systematically transforming the generating function for the values of characters associated with cycles. In the following proposition it is important to note that $l(\alpha)$ is equal to the number of cycles in each element of $C_{\alpha}$, and therefore that the number of cycles is preserved in the exponent of $z$, for future purposes.

Proposition 4.2.

$$
\sum_{\mathbf{N} \geqslant 1} \sum_{i=0}^{\mathbf{N}-1} \sum_{\alpha \vdash \mathbf{N}} \chi_{\alpha}^{\left[1^{i}, \mathbf{N}-i\right]} h^{\alpha} y^{i} z^{l(\alpha)} \frac{u^{\mathbf{N}}}{\mathbf{N} !}=(1+y)^{-1}\left\{\left(\frac{1+u y}{1-u}\right)^{z}-1\right\} .
$$

Proof. Let $\alpha=\left[1^{a_{1}} 2^{a_{2}} \cdots\right]$. Then from Corollary 3.8

$$
\sum_{i=0}^{\mathbf{N}-1} \chi_{\alpha}^{\left[\left[^{i}, \mathbf{N}-i\right]\right.} y^{i}=(1+y)^{-1} \prod_{i=1}^{\mathbf{N}}\left\{1-(-y)^{i}\right\}^{a_{i}} .
$$


But $l(\alpha)=a_{1}+\cdots+a_{\mathbf{N}}$. From Proposition 3.2, $h^{\alpha}=\mathbf{N} ! \prod_{i=1}^{\mathbf{N}}\left(i^{a_{i}} a_{i} !\right)^{-1}$. Thus

$$
\begin{aligned}
\sum_{\mathbf{N} \geqslant 1} \sum_{i=0}^{\mathbf{N}-1} \sum_{\alpha \vdash \mathbf{N}} \chi_{\alpha}^{\left[1^{i}, \mathbf{N}-i\right]} h^{\alpha} y^{i} \frac{u^{\mathbf{N}}}{\mathbf{N} !} z^{l(\alpha)} \\
=(1+y)^{-1} \sum_{\substack{a_{1}, a_{2}, \ldots \geqslant 0 \\
a_{i} \text { s not all } 0}} \prod_{i \geqslant 1} \frac{1}{a_{i} !}\left(\left\{1-(-y)^{i}\right\} z \frac{u^{i}}{i}\right)^{a_{i}} \\
=(1+y)^{-1} \prod_{i \geqslant 1} \sum_{a_{i} \geqslant 0} \frac{1}{a_{i} !}\left(\left\{1-(-y)^{i}\right\} z \frac{u^{i}}{i}\right)^{a_{i}}-(1+y)^{-1} \\
=(1+y)^{-1} \exp \left\{z \sum_{i \geqslant 1}\left\{1-(-y)^{i}\right\} \frac{u^{i}}{i}\right\}-(1+y)^{-1} .
\end{aligned}
$$

The result follows.

The following mapping is important and two of its properties are given, later, in Proposition 5.3.

Definition 4.3. Let $\phi_{z}$ be a mapping defined by $\phi_{z}\left(\begin{array}{l}z \\ k\end{array}\right)=z^{k}$, extended linearly to $R[[z]]$.

\section{LEMMA 4.4.}

$\sum_{\mathbf{N} \geqslant 1} \sum_{i=0}^{\mathbf{N}-1} \sum_{\alpha \vdash \mathbf{N}}\left(\begin{array}{c}\mathbf{N}-1 \\ i\end{array}\right)^{-1} \chi_{\alpha}^{[i, \mathbf{N}-i]} h^{\alpha} y^{i} \frac{u^{\mathbf{N}}}{\mathbf{N} !} \phi_{z} z^{l(\alpha)}=u z(1-u y z)^{-1}\{1-u(1+z)\}^{-1}$.

Proof. Let $F(y, u, z)$ denote the series

$$
\sum_{\mathbf{N} \geqslant 1} \sum_{i=0}^{\mathbf{N}-1} \sum_{\alpha \vdash \mathbf{N}} \chi_{\alpha}^{\left[1^{i}, \mathbf{N}-i\right]} h^{\alpha} y^{i} \frac{u^{\mathbf{N}}}{\mathbf{N} !} z^{l(\alpha)}
$$

so

$$
F(y, u, z)=(1+y)^{-1}\left\{\left(\frac{1+u y}{1-u}\right)^{z}-1\right\}
$$

by Proposition 4.2. But for positive integers $a, b$

$$
\int_{0}^{1} t^{a}(1-t)^{b} d t=\frac{1}{a+b+1}\left(\begin{array}{c}
a+b \\
a
\end{array}\right)^{-1}
$$

so

$$
\int_{0}^{1}(1-t)^{N}\left(\begin{array}{c}
t \\
1-t
\end{array}\right)^{m}(1-t)^{-1} d t=\frac{1}{\mathbf{N}}\left(\begin{array}{c}
\mathbf{N}-1 \\
m
\end{array}\right)^{-1}
$$

Thus

$$
\begin{aligned}
\sum_{\mathbf{N} \geqslant 1} & \sum_{i=0}^{\mathbf{N}-1} \sum_{\alpha \vdash \mathbf{N}} \frac{1}{\mathbf{N}}\left(\begin{array}{c}
\mathbf{N}-1 \\
i
\end{array}\right)^{-1} \chi_{\alpha}^{\left[\left[^{i}, \mathbf{N}-i\right]\right.} h^{\alpha} y^{i} \frac{u^{\mathbf{N}}}{\mathbf{N} !} z^{l(\alpha)} \\
& =\sum_{\mathbf{N} \geqslant 1} \sum_{i=0}^{\mathbf{N}-1} \sum_{\alpha \vdash \mathbf{N}} \chi_{\alpha}^{\left[1^{i}, \mathbf{N}-i\right]} h^{\alpha} \int_{0}^{1}\left(\frac{y t}{1-t}\right)^{i}(u(1-t))^{\mathbf{N}}(1-t)^{-1} d t z^{l(\alpha)} / \mathbf{N} ! \\
& =\int_{0}^{1} F\left(\frac{t}{1-t} y,(1-t) u, z\right)(1-t)^{-1} d t .
\end{aligned}
$$


Let

$$
G(y, u, z)=\sum_{\mathbf{N} \geqslant 1} \sum_{i=0}^{\mathbf{N}-1} \sum_{\alpha \vdash \mathbf{N}}\left(\begin{array}{c}
\mathbf{N}-1 \\
i
\end{array}\right)^{-1} \chi_{\alpha}^{\left[\left[^{i}, \mathbf{N}-i\right]\right.} h^{\alpha} y^{i} \frac{u^{\mathbf{N}}}{\mathbf{N} !} \phi_{z} z^{l(\alpha)}
$$

Then

$$
G(y, u, z)=u \frac{\partial}{\partial u} \int_{0}^{1} \phi_{z} F\left(\frac{t}{1-t} y,(1-t) u, z\right)(1-t)^{-1} d t
$$

But

$$
\begin{aligned}
\phi_{z} F(y, u, z) & =\phi_{z}(1+y)^{-1}\left\{\left(1+\frac{u(1+y)}{1-u}\right)^{z}-1\right\} \\
& =\phi_{z} \sum_{j \geqslant 1}(1+y)^{-1}\left(\begin{array}{l}
z \\
j
\end{array}\right)\left(\frac{u(1+y)}{1-u}\right)^{j} \\
& =\sum_{j \geqslant 1}\left(\frac{u z}{1-u}\right)^{j}(1+y)^{j-1}=\frac{u z}{1-u-u(1+y) z} .
\end{aligned}
$$

Thus

$$
\begin{aligned}
G(y, u, z) & =u \frac{\partial}{\partial u} \int_{0}^{1} \frac{u z d t}{1-(1-t) u-(1-t) u(1+t y /(1-t)) z} \\
& =u \frac{\partial}{\partial u} \int_{0}^{1} \frac{u z d t}{(1-u-u z)+u(1+z-y z) t} \\
& =u \frac{\partial}{\partial u} \frac{z}{1+z(1-y)} \cdot \log \frac{1-u y z}{1-u(1+z)}
\end{aligned}
$$

and the result follows.

We may now give the main theorem.

THEOREM 4.5.

$$
z+\sum_{k, \mathbf{N} \geqslant 1} \sum_{\langle\mathbf{a}\rangle \vdash \mathbf{N}} e_{k}^{\langle\mathbf{a}\rangle} \mathbf{w}^{\mathbf{a}} \frac{u^{\mathbf{N}}}{\mathbf{N} !} \phi_{z} z^{k}=z \exp \left\{\sum_{i \geqslant 1} \frac{1}{i}\left\{(1+z)^{i}-z^{i}\right\} u^{i} w_{i}\right\} .
$$

Proof. Let $\langle a\rangle \vdash$ N. Then from Proposition 4.1

$$
\sum_{\alpha \vdash \mathbf{N}} e_{\alpha}^{\langle\mathbf{a}\rangle} \phi_{z} z^{l(\alpha)}=\frac{1}{\mathbf{N} !} h^{\langle\mathbf{a}\rangle} \sum_{i=0}^{\mathbf{N}-1} \sum_{\alpha \vdash \mathbf{N}}(-1)^{i}\left(\begin{array}{c}
\mathbf{N}-1 \\
i
\end{array}\right)^{-1} h^{\alpha} \chi_{\alpha}^{\left[\left[^{i}, \mathbf{N}-i\right]\right.} \chi_{\langle\mathbf{a}\rangle}^{\left[1^{i}, \mathbf{N}-i\right]} \phi_{z} z^{l(\alpha)}
$$

so, from Corollary 3.8 and Lemma 4.4

$$
\begin{aligned}
\sum_{\alpha \vdash \mathbf{N}} e_{\alpha}^{\langle\mathbf{a}\rangle} \phi_{z} z^{l(\alpha)}= & h^{\langle\mathbf{a}\rangle} \sum_{j=0}^{\mathbf{N}-1}\left\{\left[u^{N} y^{j}\right] u z(1-u y z)^{-1}\{1-u(1+z)\}^{-1}\right\} \\
& \cdot\left\{\left[y^{j}\right](1-y)^{-1} \prod_{i \geqslant 1}\left(1-y^{i}\right)^{a_{i}}\right\} .
\end{aligned}
$$

But

$$
\left[u^{\mathbf{N}} y^{j}\right] u z(1-u y z)^{-1}\{1-u(1+z)\}^{-1}= \begin{cases}z^{j+1}(1+z)^{\mathbf{N}-j-1} & \text { if } 0 \leqslant j<\mathbf{N} \\ 0 & \text { otherwise }\end{cases}
$$


so

$$
\begin{aligned}
\sum_{\alpha \vdash \mathbf{N}} e_{\alpha}^{\langle\mathbf{a}\rangle} \phi_{z} z^{l(\alpha)} & =h^{\langle\mathbf{a}\rangle} z(1+z)^{\mathbf{N}-1} \sum_{j=0}^{\mathbf{N}-1} z^{j}(1+z)^{-j}\left[y^{j}\right](1-y)^{-1} \prod_{i \geqslant 1}\left(1-y^{i}\right)^{a_{i}} \\
& =h^{\langle\mathbf{a}\rangle} z(1+z)^{\mathbf{N}} \prod_{i \geqslant 1}\left\{1-\left(\frac{z}{1+z}\right)^{i}\right\}^{a_{i}} .
\end{aligned}
$$

Thus, from Proposition 3.2(i)

$$
\sum_{\alpha \vdash \mathbf{N}} e_{\alpha}^{\langle\mathbf{a}\rangle} \phi_{z} z^{l(\alpha)} \frac{u^{\mathbf{N}}}{\mathbf{N} !} \mathbf{w}^{\mathbf{a}}=z \prod_{i \geqslant 1} \frac{1}{a_{i} !}\left\{\frac{1}{i} u^{i} w_{i}\left\{(1+z)^{i}-z^{i}\right\}\right\}^{a_{l}}
$$

since $\mathbf{N}=\sum_{i} i a_{i}$. The result follows by summing over the $a_{i}$.

As an immediate consequence of this theorem we can give an explicit expression for $e_{k_{0}}^{\gamma}$ when $k_{0}$ is the largest number of cycles in any of the elements of $C_{[\mathbf{N}]} C_{\gamma}$, where $\gamma \vdash \mathbf{N}$.

Corollary 4.6. Let $k_{0}(\langle\mathbf{a}\rangle)=\max _{k}\left\{k \mid e_{k}^{\langle\mathbf{a}\rangle} \neq 0\right\}$, where $\langle\mathbf{a}\rangle \vdash \mathbf{N}$. Then

(i) $k_{0}(\langle\mathrm{a}\rangle)=\mathbf{N}+1-\sum_{i} a_{i}$

(ii) $e_{k_{0}}^{\langle\mathbf{a}\rangle}=\mathbf{N} ! /\left(\mathbf{N}+1-\sum_{i} a_{i}\right) ! \prod_{i} a_{i} !$

Proof. From Theorem 4.5

$$
\begin{aligned}
\sum_{k} e_{k}^{\langle\mathbf{a}\rangle} z^{k} & =\mathbf{N} ! \phi_{z}^{-1} z \prod_{i}\left\{(1+z)^{i}-z^{i}\right\}^{a_{i}} / a_{i} ! i^{a_{i}} \\
& =\frac{\mathbf{N} !}{\prod_{i} a_{i} !} \phi_{z}^{-1} z^{\mathbf{N}+1-\sum_{i} a_{i}}+O\left(z^{\mathbf{N}-\sum_{i} a_{i}}\right) \\
& =\frac{\mathbf{N} !}{\prod_{i} a_{i} !\left(\mathbf{N}+1-\sum_{i} a_{i}\right) !} z^{\mathbf{N}+1-\sum_{i} a_{i}}+O\left(z^{\mathbf{N}-\sum_{i} a_{i}}\right)
\end{aligned}
$$

and the results follow.

We return to this result in $\$ 7$, where it is proved by a direct combinatorial argument.

5. Explicit forms for special cases. It is possible to obtain an explicit expression for $e_{k}^{(p)}(n)$ in terms of some well-known combinatorial numbers.

Definition 5.1. The Stirling numbers of the first and second kinds are given, respectively, by

(i)

$$
\frac{1}{m !}\{\log (1+x)\}^{m}=\sum_{n=m}^{\infty} s_{n}^{(m)} \frac{x^{n}}{n !}
$$

(ii)

$$
\frac{1}{m !}\left(e^{x}-1\right)^{m}=\sum_{n=m}^{\infty} S_{n}^{(m)} \frac{x^{n}}{n !} .
$$

An elementary combinatorial argument shows that $s_{n}^{(m)}$ is $(-1)^{n-m}$ times the number of permutations in $\mathbf{S}_{n}$ with exactly $m$ cycles, and that $S_{n}^{(m)}$ is the number of partitions of $[n]$ into exactly $m$ nonempty blocks. 
The following properties are immediate.

Proposition 5.2. (i)

(ii)

$$
\left(\begin{array}{l}
x \\
n
\end{array}\right)=\frac{1}{n !} \sum_{m=0}^{n} s_{n}^{(m)} x^{m}
$$

$$
x^{n}=\sum_{m=0}^{n} m ! S_{n}^{(m)}\left(\begin{array}{l}
x \\
m
\end{array}\right)
$$

(iii)

$$
S_{n}^{(m)}=\frac{1}{m !} \sum_{j=0}^{m}(-1)^{m-j}\left(\begin{array}{c}
m \\
j
\end{array}\right) j^{n} .
$$

The next result concerns the action of $\phi_{z}$, defined in Definition 4.3, on certain polynomials in $z$.

PROPOSITION 5.3. (i) $\phi_{z} z^{n}=\sum_{m=0}^{n} m ! S_{n}^{(m)} z^{m}$.

(ii) $\phi_{z}^{-1} z^{k}(1+z)^{l}=\left(\begin{array}{c}z+l \\ k+l\end{array}\right)$.

Proof. (i) Immediate consequence of Proposition 5.2(ii).

(ii)

$$
\phi_{z}^{-1} z^{k}(1+z)^{l}=\sum_{j=0}^{l}\left(\begin{array}{l}
l \\
j
\end{array}\right) \phi_{z}^{-1} z^{k+j}=\sum_{j=0}^{l}\left(\begin{array}{l}
l \\
j
\end{array}\right)\left(\begin{array}{c}
z \\
k+j
\end{array}\right)=\left(\begin{array}{l}
z+l \\
k+l
\end{array}\right) .
$$

\section{THEOREM 5.4 .}

$$
e_{k}^{(p)}(n)=\frac{1}{(1+p n) p^{n+k}} \sum_{m=n+k}^{1+p n} p^{m}\left(\begin{array}{c}
m \\
k
\end{array}\right) s_{p n+1}^{(m)} S_{m-k}^{(n)} .
$$

Proof. From Theorem 4.5

$$
\begin{aligned}
e_{k}^{(p)}(n) & =\frac{(p n) !}{p^{n} n !}\left[z^{k}\right] \phi_{z}^{-1} z\left\{(1+z)^{p}-z^{p}\right\}^{n} \\
= & \frac{(p n) !}{p^{n} n !}\left[z^{k}\right] \phi_{z}^{-1} \sum_{j=0}^{n}\left(\begin{array}{c}
n \\
j
\end{array}\right)(-1)^{n-j}(1+z)^{j p} z^{(n-j) p+1} \\
= & (-1)^{n} \frac{(p n) !}{p^{n} n !}\left[z^{k}\right] \sum_{j=0}^{n}(-1)^{j}\left(\begin{array}{c}
n \\
j
\end{array}\right)\left(\begin{array}{c}
z+j p \\
1+n p
\end{array}\right) \quad(\text { from Proposition 5.3(ii)) } \\
= & (-1)^{n} \frac{(p n) !}{p^{n} n !} \sum_{j=0}^{n}(-1)^{j}\left(\begin{array}{l}
n \\
j
\end{array}\right) \frac{1}{(1+p n) !} \sum_{m=0}^{p n+1} s_{p n+1}^{(m)}\left[z^{k}\right](z+j p)^{m} \\
= & (-1)^{n} \frac{1}{p^{n} n !} \sum_{j=0}^{n}(-1)^{j}\left(\begin{array}{l}
n \\
j
\end{array}\right) \frac{1}{(1+p n)} \sum_{m=0}^{p n+1} s_{p n+1}^{(m)}\left(\begin{array}{c}
m \\
k
\end{array}\right)(j p)^{m-k} \\
= & \frac{(-1)^{n}}{n ! p^{n+k}(1+p n)} \cdot \sum_{m=0}^{1+p n}\left(\begin{array}{c}
m \\
k
\end{array}\right) p^{m} s_{p n+1}^{(m)} \sum_{j=0}^{n}(-1)^{j}\left(\begin{array}{c}
n \\
j
\end{array}\right) j^{m-k}
\end{aligned}
$$

and the result follows from Proposition 5.2(iii). 
Simple expressions for $e_{k}^{(p)}(n)$ for values of $k$ close to $k_{0}\left(\left[p^{n}\right]\right)$ may be obtained directly from this theorem. From Corollary 4.6(i), $k_{0}\left(\left[p^{n}\right]\right)=n(p-1)+1$.

Corollary 5.5. (i)

$$
e_{n(p-1)+1}^{(p)}(n)=\frac{1}{n(p-1)+1}\left(\begin{array}{c}
p n \\
n
\end{array}\right),
$$

(ii)

$$
e_{n(p-1)-1}^{(p)}(n)=\frac{1}{12} n p\left\{n\left(\begin{array}{c}
p \\
2
\end{array}\right)-1\right\}\left(\begin{array}{c}
n p-1 \\
n
\end{array}\right) .
$$

Proof. From Definition 5.1

$$
\begin{array}{cll}
s_{(n)}^{(n)}=1, & S_{n+1}^{(n)}=-\frac{1}{2} n(n+1), & s_{n+2}^{(n)}=\frac{1}{24} n(n+1)(n+2)(3 n+5), \\
S_{n}^{(n)}=1, & S_{n}^{(n-1)}=\frac{1}{2} n(n-1), & S_{n}^{(n-2)}=\frac{1}{24} n(n-1)(n-2)(3 n-5) .
\end{array}
$$

The results follow from Theorem 5.4.

Thus, $e_{n+1}^{(2)}(n)$ is a Catalan number.

6. Recurrence equations. Theorem 4.5 can be used to obtain further properties of the sequence $\left\{e_{k}^{(p)}(n) \mid k, n \geqslant 1\right\}$.

LEMMA 6.1. (i) $e_{k}^{(2)}(n)=0$ if $n+1-k$ is odd,

(ii) $e_{k}^{(2)}(n)$ satisfies the recurrence equation

$$
(n+1) e_{k}^{(2)}(n)=(2 n-1)(n-1)(2 n-3) e_{k}^{(2)}(n-2)+2(2 n-1) e_{k-1}^{(2)}(n-1)
$$

with boundary conditions

$$
\begin{aligned}
& \text { (a) } e_{k}^{(2)}(n)=0 \quad \text { if } k \leqslant 0, \\
& \text { (b) } e_{k}^{(2)}(n)= \begin{cases}0 & \text { if } n<0, \\
\delta_{k 1} & \text { if } n=0 .\end{cases}
\end{aligned}
$$

Proof. From Theorem 4.5

$$
\begin{aligned}
z+\sum_{n \geqslant 1} \sum_{k} \frac{n !}{(2 n) !} e_{k}^{(2)}(n) u^{n} z^{k} & =\phi_{z}^{-1} z\left\{1-\frac{u}{2}\left\{(1+z)^{2}-z^{2}\right\}\right\}^{-1} \\
& =\left(1-\frac{u}{2}\right)^{-1} \phi_{z}^{-1} z\left\{1-\left(1-\frac{u}{2}\right)^{-1} u z\right\}^{-1} \\
& =\left(1-\frac{u}{2}\right)^{-1} \sum_{m \geqslant 0}\left(1-\frac{u}{2}\right)^{-m} u^{m}\left(\begin{array}{c}
z \\
m+1
\end{array}\right)
\end{aligned}
$$

so

$$
1+u z+\sum_{n \geqslant 1} \sum_{k \geqslant 1} \frac{n !}{(2 n) !} e_{k}^{(2)}(n) u^{n+1} z^{k}=\left(1+\frac{u}{2}\right)^{z}\left(1-\frac{u}{2}\right)^{-z} \in \mathbf{C}[z][[u]] .
$$

Let $z$ be replaced by $z^{-1}$, and $u$ by $u z$ so

$$
1+u+\sum_{n \geqslant 1} \sum_{k \geqslant 1} \frac{n !}{(2 n) !} e_{k}^{(2)}(n) u^{n+1} z^{n+1-k}=\left(1+\frac{u z}{2}\right)^{1 / z} \cdot\left(1-\frac{u z}{2}\right)^{-1 / z} .
$$


Let $F(u, z)$ denote the right-hand side of this equation. Then $F(u,-z)=F(u, z)$ so $F$ is an even Laurent series in $z$. Thus $e_{k}^{(2)}(n)=0$ if $n+1-k$ is odd and (i) follows. Evidently $F$ satisfies the formal differential equation $\left(1-z^{2} u^{2} / 4\right) \partial F / \partial u=$ $F$. The recurrence equation then follows.

Lemma 6.1(ii) can be used to calculate particular values of $e_{k}^{(2)}(n)$. For example, with $k=1$,

$$
e_{1}^{(2)}(n)=\frac{1}{n+1}(2 n-1)(n-1)(2 n-3) e_{1}^{(2)}(n-2),
$$

where $e_{1}^{(2)}(1)=0, e_{1}^{(2)}(0)=1$ so

$$
e_{1}^{(2)}(n)= \begin{cases}\frac{1}{2^{n}} \frac{(2 n) !}{(n+1) !} & \text { if } n \text { is even } \\ 0 & \text { if } n \text { is odd. }\end{cases}
$$

LEMMA 6.2. $\left\{e_{k}^{(3)}(n) \mid k, n \geqslant 0\right\}$ satisfies a linear recurrence equation with coefficients in $\mathbf{C}[k, n]$.

Proof. From Theorem 4.5

$$
\begin{aligned}
z+\sum_{n \geqslant 1} \sum_{k=1}^{2 n+1} n ! e_{k}^{(3)} & (n) \frac{u^{n}}{(3 n) !} z^{k}=\phi_{z}^{-1} z\left\{1-\frac{u}{3}\left\{(1+z)^{3}-z^{3}\right\}\right\}^{-1} \\
& =\phi_{z}^{-1} z\left(1-\frac{u}{3}\right)^{-1}\left\{1-\left(1-\frac{u}{3}\right)^{-1} u z(1+z)\right\}^{-1} \\
& =\sum_{k \geqslant 0} u^{k}\left(1-\frac{u}{3}\right)^{-(k+1)}\left(\begin{array}{c}
z+k \\
2 k+1
\end{array}\right) \quad \text { (from Proposition 5.3(ii)) } \\
& =\left(1-\frac{u}{3}\right)^{-1} \sum_{k \geqslant 0} u^{k}\left(1-\frac{u}{3}\right)^{-k} \frac{(z+k)_{(2 k+1)}}{(2 k+1) !}
\end{aligned}
$$

where $(m)^{(r)}=m(m+1) \cdots(m+r-1)$ and $(m)_{(r)}=m(m-1) \cdots(m-r+1)$. But

$$
(z+k)_{(2 k+1)}=(-1)^{k}(z+1)^{(k)} z(1-z)^{(k)} ;(1+2 k) !=4^{k} k !\left(\frac{3}{2}\right)^{(k)}
$$

so

$z+\sum_{n \geqslant 1} \sum_{k=1}^{2 n+1} n ! e_{k}^{(3)}(n) \frac{u^{n}}{(3 n) !} \cdot z^{k}=z\left(1-\frac{u}{3}\right)^{-1}{ }_{2} F_{1}\left[\begin{array}{c}1+z, 1-z \\ \frac{3}{2}\end{array} ; \frac{-u}{4}\left(1-\frac{u}{3}\right)^{-1}\right]$,

where

$$
{ }_{2} F_{1}[\underset{c}{a, b} ; v]=\sum_{n \geqslant 0} \frac{(a)^{(n)}(b)^{(n)}}{(c)^{(n)}} \frac{v^{n}}{n !}
$$

is a hypergeometric series. But this satisfies Gauss' equation (Slater [6])

$$
v(v-1) \frac{d^{2} y}{d v^{2}}+\{c-(1+a+b) v\} \frac{d y}{d v}-a b v=0 .
$$


Thus by change of variable

$$
\left(1-\frac{u}{3}\right)\left\{z+\sum_{n \geqslant 1} \sum_{k=1}^{2 n+1} n ! e_{k}^{(3)}(n) \frac{u^{n}}{(3 n) !} z^{k}\right\}
$$

satisfies a differential equation in $u$ with coefficients which are polynomials in $u$ and $z$. The result follows.

LEMMA 6.3. For $r \geqslant 1$

$$
e_{n+1-2 r}^{(2)}(n)=\frac{(2 n) !}{n !(n-2 r) !} \cdot \frac{1}{(2(r+1)) !} \rho_{r}(n),
$$

where $\rho_{r}(n)$ is a polynomial of degree $r-1$ in $n$.

Proof. We use induction over $r \geqslant 1$. Let

$$
e_{n+1-2 r}^{(2)}(n)=\frac{(2 n) !}{n !(n-2 r) !} \frac{1}{(2(r+1)) !} \rho_{r}(n) .
$$

Then, from Lemma 6.1(ii)

$$
(n+1) \rho_{r}(n)=(n-2 r) \rho_{r}(n-1)+\frac{1}{2}(n-1)(r+1)(2 r+1) \rho_{r-1}(n-2),
$$

where $\rho_{0}(0)=2, \rho_{r}(n)=0$ if $n+1-2 r \leqslant 0$, and $\rho_{r}(n)=0$ if $n<0$. Regard $r$ as fixed. Now the general solution of this recurrence equation is

$$
\rho_{r}(n)=\theta_{r}(n)+P_{r}(n),
$$

where $\theta_{r}(n)$ is the general solution of

$$
(n+1) u_{r}(n)=(n-2 r) u_{r}(n-1)
$$

and $P_{r}(n)$ is a solution of the recurrence equation for $\rho_{r}(n)$. Clearly,

$$
\theta_{r}(n)=\frac{(2 r+1) !}{(n+1)(n+2) \cdots(n-2 r+1)} \theta_{r}(2 r) \text { for } n \geqslant 2 r
$$

so $\theta_{r}(n)=0$ for $n \geqslant 2 r$ since $\theta_{r}(2 r)=0$.

Now suppose that $\rho_{r-1}(n)$ is a polynomial of degree $r-2$ in $n$. Then

$$
(n+1) P_{r}(n)-(n-2 r) P_{r}(n-1)=\frac{1}{2}(r+1)(2 r+1)(n-1) \rho_{r-1}(n-2)
$$

which is a polynomial of degree $r-1$ in $n$. Thus

$$
n\left\{P_{r}(n)-P_{r}(n-1)\right\}+P_{r}(n)+2 r P_{r}(n-1)
$$

is a polynomial of degree $r-1$ in $n$. For a particular solution suppose

$$
P_{r}(n)=\sum_{j=0}^{r-1} a_{j}^{(r)} n^{j}
$$

so $n\left(P_{r}(n)-P_{r}(n-1)\right)$ is a polynomial of degree $r-1$ in $n$. The values of $a_{j}^{(r)}$ are uniquely determined by comparing coefficients of $n^{j}$ for $j=0,1, \ldots, r-1$. Thus $\rho_{r}(n)$ is a polynomial of degree $r-1$ in $n$. Finally, from Corollary 5.5(ii),

$$
e_{n-1}^{(2)}(n)=\frac{1}{3 !} n(n-1)\left(\begin{array}{c}
2 n-1 \\
n
\end{array}\right)
$$

so $\rho_{1}(n)=2$, a polynomial of degree 0 in $n$. The result now follows. 
Observe from the proof of Lemma 6.3 that the coefficients of $\rho_{r}(n)$ satisfy a linear recurrence equation. These coefficients are given in Table II of the Appendix.

7. Combinatorial remarks. Clearly, it would be of considerable interest if the expression for $e_{k}^{\gamma}$ could be proved combinatorially, without direct appeal to properties of $\mathbf{C S}_{\mathbf{N}}$. As a special case, consider $e_{k_{0}}^{\gamma}$, where $\gamma=\left[1^{a_{1}} 2^{a_{2}} \ldots\right] \vdash \mathbf{N}$ and where $k_{0}$ is the largest number of cycles possessed by any element of $C_{[\mathrm{N}]} C_{\gamma}$. Such a permutation can be represented by a dissection of a convex $\mathbf{N}$-gon in the plane by $a_{i}$ $i$-gons for $i=1,2, \ldots$, which are vertex and edge disjoint. The orientation of each polygon is uniquely determined. The number of such dissections is $e_{k_{0}}^{\gamma}$. Let

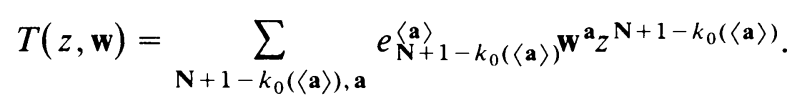

Then an elementary combinatorial argument shows that $T$ must satisfy the functional equation $T=1+z \sum_{i \geqslant 1} T^{i} w_{i}$. Let $S=T-1$ so $S=z \sum_{i \geqslant 1}(S+1)^{i} w_{i}$, whence, by Lagrange's Theorem

$$
\begin{aligned}
e_{\mathbf{N}+1-k_{0}(\langle\mathbf{a}\rangle)}^{\gamma} & =\left[z^{\mathbf{N}+1-k_{0}(\langle\mathbf{a}\rangle)} \mathbf{w}^{\mathbf{a}}\right] S \\
& =\frac{1}{\mathbf{N}+1-k_{0}(\langle\mathbf{a}\rangle)}\left[\lambda^{\mathbf{N}+1-k_{0}(\langle\mathbf{a}\rangle)-1}\right](\lambda+1)^{\mathbf{N}} \frac{\left(\sum a_{i}\right) !}{\prod_{i} a_{i} !}=\frac{\mathbf{N} !}{k_{0} ! \Pi_{i} a_{i} !},
\end{aligned}
$$

which is the statement of Corollary 4.6(ii).

Theorem 4.5 may be used to obtain further combinatorial information about $e_{k}^{\gamma}$. We shall say that a permutation is dotted if each element in the cycles of the permutation has associated with it at most one dot. This is done by placing the dot, if it occurs, above the corresponding element. Thus $(1 \dot{2})(\dot{3} 4)$ is a dotted permutation. A permutation is strictly dotted if none of its cycles has all of its elements bearing a dot, and a dotted permutation has weight $m$ if it has a total of $m$ dots.

From Theorem 4.5 and Proposition 5.3(i)

$$
\sum_{k} e_{k}^{\gamma} \cdot m ! S_{k}^{(m)}=\left[z^{m-1} \frac{u^{\mathbf{N}}}{\mathbf{N} !} \mathbf{w}^{\mathbf{a}}\right] \exp \sum_{i} \frac{u^{i}}{i} w_{i}\left\{(1+z)^{i}-z^{i}\right\} .
$$

But the left-hand side is the number of ways of partitioning the cycles of permutations, counted by $e_{k}^{\gamma}$, into $m$ nonempty ordered blocks. This is a consequence of the comment following Definition 5.1. For convenience let us call such permutations ordered partition permutations of weight $m$ in $\omega_{\mathrm{N}} C_{\gamma}$. Moreover, by an elementary combinatorial argument, the right-hand side is the number of strictly dotted permutations in $C_{\gamma}$ of weight $m-1$.

Thus, from the above equation, we have shown that the following result is a combinatorial restatement of Theorem 4.5.

THEOREM 7.1 Let $\gamma \vdash \mathbf{N}$. Then the number of ordered partition permutations of weight $m$ in $\omega_{\mathrm{N}} C_{\gamma}$ is equal to the number of strictly dotted permutations of weight $m-1$ in $C_{\gamma}$ for $m \geqslant 1$.

A purely combinatorial proof of this bijection would establish Theorem 4.5, and would be of considerable interest. 
ACKNOWLEDGMENT. The author thanks R. P. Stanley for bringing this problem to his attention. The work was carried out while the author was a Visiting Scholar in the Mathematics Department at MIT from August 1984 to June 1985. The author acknowledges a Travel Grant from the Natural Sciences and Engineering Research Council of Canada.

Appendix.

TABLE I. $e_{n+1-2 g}^{(2)}(n), 1 \leqslant n \leqslant 10 ; 0 \leqslant g \leqslant\lfloor n / 2\rfloor$.

\begin{tabular}{cllllll}
$n \backslash g$ & \multicolumn{1}{c}{0} & \multicolumn{1}{c}{1} & \multicolumn{1}{c}{2} & \multicolumn{1}{c}{3} & \multicolumn{1}{c}{4} & 5 \\
1 & 1 & & & & & \\
2 & 2 & 1 & & & & \\
3 & 5 & 10 & & & & \\
4 & 14 & 70 & 21 & & & \\
5 & 42 & 420 & 483 & & & \\
6 & 132 & 2310 & 6468 & 1485 & & \\
7 & 429 & 12012 & 66066 & 56628 & & \\
8 & 1430 & 60060 & 570570 & 1169740 & 225225 & \\
9 & 4862 & 291720 & 4390386 & 17454580 & 12317877 & \\
10 & 16796 & 1385670 & 31039008 & 211083730 & 351683046 & 59520825
\end{tabular}

TABLE II. Tabulation of $b_{i}^{(r)}$ for $1 \leqslant r \leqslant 6$, where

\begin{tabular}{|c|c|c|c|c|c|c|c|}
\hline$\rho_{r}(n)$ & $=c \sum_{i \geqslant}$ & ${ }_{i}^{(r)} n^{i}$ an & $e_{n+1-2 r}^{(2)}$ & $n)=\frac{1}{2}(n+$ & $1)^{-1}(r+$ & )$^{-1}\left(\begin{array}{c}2 n \\
n\end{array}\right)\left(\begin{array}{c}n \\
2 r\end{array}\right.$ & $\left.\begin{array}{l}+1 \\
+1\end{array}\right) \rho_{r}(n)$. \\
\hline$r$ & $c$ & $i=0$ & 1 & 2 & 3 & 4 & 5 \\
\hline 1 & 1 & 2 & & & & & \\
\hline 2 & $\frac{1}{2}$ & -2 & 5 & & & & \\
\hline 3 & $\frac{1}{9}$ & 12 & -77 & 35 & & & \\
\hline 4 & $\frac{1}{24}$ & -72 & 1094 & -945 & 175 & & \\
\hline 5 & $\frac{1}{24}$ & 240 & -8954 & 11099 & -3850 & 385 & \\
\hline 6 & $\frac{1}{4320}$ & -199008 & 19419660 & -30398368 & 14899885 & -2802800 & 175175 \\
\hline
\end{tabular}

\section{REFERENCES}

1. M. Burrow, Representation theory of finite groups, Academic Press, New York, 1965.

2. Private communication.

3. J. Harer and D. Zagier, The Euler characteristic of the moduli space of curves, Invent. Math. 85 (1986), 457-485. 
4. J. Harris and I. Morrison, Slopes of effective divisors on the moduli space of stable curves, (preprint).

5. I. G. Macdonald, Symmetric functions on Hall polynomials, Clarendon Press, Oxford, 1979.

6. L. J. Slater, Generalized hypergeometric functions, Cambridge Univ. Press, Cambridge, 1966.

7. R. P. Stanley, Factorization of permutations into n-cycles, Discrete Math. 37 (1981), 255-262.

8. J. G. Thompson, Rational functions associated to presentations of finite groups, J. Algebra 71 (1981), 481-489.

Department OF COMbinatorics and Optimization, University of Waterloo, Waterloo, Ontario, Canada N2L 3G1 